Revue européenne des sciences sociales

European Journal of Social Sciences

XL-124 | 2002

Histoire, philosophie et sociologie des sciences

\title{
La connaissance a-t-elle un sujet ? Un essai pour repenser l'individu
}

\section{Catherine Chevalley}

\section{(2) OpenEdition}

\section{Journals}

Édition électronique

URL : http://journals.openedition.org/ress/570

DOI : $10.4000 /$ ress. 570

ISSN : 1663-4446

Éditeur

Librairie Droz

Édition imprimée

Date de publication : 1 août 2002

Pagination : 7-26

ISBN : 2-600-00806-3

ISSN : 0048-8046

Référence électronique

Catherine Chevalley, « La connaissance a-t-elle un sujet ? Un essai pour repenser l'individu », Revue européenne des sciences sociales [En ligne], XL-124 | 2002, mis en ligne le 01 décembre 2009, consulté le 10 décembre 2020. URL : http://journals.openedition.org/ress/570 ; DOI : https://doi.org/10.4000/ ress.570 


\section{LA CONNAISSANCE A-T-ELLE UN SUJET? UN ESSAI POUR REPENSER L'INDIVIDU}

Il y a sans doute en philosophie aujourd'hui peu de questions d'allure aussi classiques et académiques que celle du sujet. Pourtant, lorsqu'il s'agit de traiter des rapports entre philosophie, histoire des sciences et sociologie des sciences, le problème que pose la détermination d'un concept de sujet est généralement éludé; ou bien, ce qui produit le même néant de résultats, il est phagocyté par la polémique ou noyé dans l'œcuménisme. Je voudrais donc procéder autrement et affronter directement la difficulté de savoir comment l'on peut se former aujourd'hui un concept à peu près rigoureux de l'individu qui connaît, de l'individu que je suis moi-même en tant qu'engagé dans l'activité de comprendre.

Pour cela j'adopterai le parti d'opposer à une première figure possible du sujet, celle qui l'identifie plus ou moins à la pensée collective d'une communauté, une seconde figure, caractérisée par l'invention de techniques individuelles d'écart à l'égard de cette pensée collective et par la stylisation d'une conduite nouvelle en réponse à des situations de contradiction. En introduisant à propos de la connaissance l'idée qu'un individu mis, pour une raison ou pour une autre, «au pied du mur» s'enseigne à lui-même des automatismes nouveaux, je voudrais faire apparaître l'idée que comprendre, c'est inventer et que c'est aussi, simultanément, s'inventer soi-même.

Je généraliserai ensuite cette seconde figure en disant que l'individu qui se constitue comme sujet de ses actes dans la pratique cognitive fait de cette manière l'une des expériences fondamentales qui caractérisent ce que M. Foucault appelait le mode de subjectivation. Ainsi devient-il possible de penser la connaissance comme un processus de fabrication de soi. Ni «participation» platonicienne, ni décodage de lois universelles et nécessaires au sens de Galilée, ni pouvoir baconien, ni empilement d'habitudes et d'associations, ni savante mise en branle des rouages de mon entendement, ni simple domestication de la peur, ni négociation sociale des preuves. Comprendre n'a de sens pour l'individu, en dernière instance, que comme une pratique de soi qui ouvre à un art de «se gouverner soi-même et les autres».

\section{SOCIOLOGIE DES SCIENCES : VIVE LA COMMUNAUTÉ}

Par sujet, on a entendu en général dans la philosophie moderne une façon de désigner l'individu comme le support de pensées, de représentations, de perceptions, d'imaginations. On a dit souvent que cette façon de faire était calquée sur la structure apophantique-formelle du sujet grammatical. Dans le langage ordinaire, 
on nomme sujet ce qui reçoit des prédicats, et de la même manière Descartes aurait pensé l'ego cogito comme ce qui est le fondement de cogitationes. Kant aurait conservé la même orientation fondamentale, et lorsque Husserl en vient à s'exprimer sur la question, dans les années 1930, c'est pour dire que toute la philosophie moderne s'est emprisonnée dans le problème absurde de savoir comment le Moi, avec ses vécus intentionnels, pouvait sortir de lui-même et entrer en relation avec le monde des objets et prétendre le connaître.

Il se trouve que, dans ces années 1930, le sujet connaissant se voit partout délogé de ses forteresses traditionnelles et remplacé par autre chose, dont la dénomination varie selon les auteurs. Du côté de la sociologie, cet autre chose s'appelle la communauté. Je rappellerai donc pour commencer les grandes lignes de l'opération tentée par $\mathrm{L}$. Fleck ${ }^{1}$ pour soutenir que l'agent réel de la connaissance serait le Penser-collectif (Denkkollektiv) de la communauté. Qui connaît? C'est la communauté. Comment? En transmettant des techniques, des savoirs-faire, des concepts, des lexiques, des uniformités de perception, etc. L'expulsion explicite de toute philosophie possible de la connaissance réflexive que décrète Fleck, comme beaucoup d'autres à cette époque, installe la pensée dans l'ordre du collectif et de l'anonyme. Voyons comment un peu plus en détail.

\section{A) La notion de Penser-collectif chez L. Fleck}

La connaissance est une activité sociale de part en part. Cette affirmation est martelée par Fleck, dans son livre Genèse et développement d' un fait scientifique ${ }^{2}$, dans l'intention de fonder un nouveau genre de théorie de la connaissance, qu'il nomme épistémologie comparative. Fleck veut établir que la connaissance n'est pas un «processus individuel relevant d'une quelconque conscience théorétique » ${ }^{3}$ et que le mot même de connaissance n'a de sens qu'en rapport avec l'idée d'un

\footnotetext{
L. Fleck a été l'inspirateur direct de T. S. Kuhn. Né en Pologne en 1896, médecin, il dirigeait le laboratoire de bactériologie de l'hôpital de Lvov quand il est renvoyé en 1935 à la suite des mesures prises contre les juifs. De 1935 à 1939, Fleck travaille à Lvov dans son laboratoire privé de microbiologie et continue ses recherches sur les streptocoques. Il découvre une méthode nouvelle pour renforcer l'efficacité de la réaction de Wassermann. Après l'entrée des Soviétiques à Lvov, il fait partie du corps enseignant de la Faculté de Médecine, en même temps qu'il est consultant en immunologie et sérologie. Puis, sous l'occupation allemande à partir de 1941, Fleck travaille à l'Hôpital juif, où il doit faire face à l'épidémie de typhus dans le ghetto. Arrêté en 1942, déporté à Auschwitz en 1943, rattaché à l'hôpital du camp, il est transféré à Buchenwald. A la libération du camp en 1945, Fleck rentre en Pologne, où il recommence à enseigner. Il est témoin à Nüremberg en 1948. En 1950, il devient professeur à Lublin et, en 1952, à Varsovie. Il meurt en 1961.

2 L. Fleck, Entstehung und Entwicklung einer wissenschaftlichen Tatsache: Einführung in die Lehre vom Denkstil und Denkkollektiv, Bâle, Benno Schwabe \& Co. Ce livre est peu connu dans le détail de son contenu; je citerai ici le texte de sa traduction anglaise par T. Trenn, R. K. Merton et F. Bradle, Genesis and Development of a Scientific Fact (avec une Préface de T. S. Kuhn), University of Chicago Press, Londres et Chicago, 1979 (abrégé en Genesis). Pour l'affirmation que la connaissance est une activité fondamentalement sociale, cf. ibid., resp. p. 42 et p. 98: «Cognition is the most socially-conditioned activity of man, and knowledge is the paramount social creation (...) I (call) thinking a supremely social activity which cannot by any means be completely localized within the confines of the individual».

3 L. Fleck, Genesis, p. 38.
} 
Penser-collectif ${ }^{4}$. Défini comme «une communauté de personnes qui échangent des idées ou qui sont en interaction intellectuelle de manière continue», ce Penser-collectif ne doit pas être conçu comme un agrégat d'individus ${ }^{5}$; il s'agit plutôt d'un monde de pensée autonome et global qui fournit le fond du savoir existant,

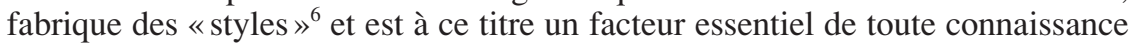
nouvelle.

Le Penser-collectif fournit à Fleck le moyen de briser la relation duelle entre sujet et objet qui caractérise à ses yeux toutes les conceptions de la connaissance dans la philosophie moderne. D'une relation de lecture, décodage, traduction, reflet ou expression entre sujet et objet, la connaissance devient alors un processus à trois éléments: l'individu, les états de choses et le Penser-collectif:

\begin{abstract}
In comparative epistemology, cognition must not be construed as only a dual relationship between the knowing subject and the object to be known. The existing fund of knowledge must be a third partner in this relation as a basic factor of all new knowledge. It would otherwise remain beyond our understanding how a closed and style-permeated system of opinions could arise, and why we find, in the past, rudiments of current knowledge which at the time could not be legitimized by any «objective» reasons and which remained only pre-ideas ${ }^{7}$.
\end{abstract}

L. Fleck, Genesis, p. 43.

5 L. Fleck, Genesis, p. 40: «Although the Thought collective consists of individuals, it is not simply the agregate sum of them ».

6 L. Fleck utilise abondamment la notion de «style de pensée» (Denkstil), déjà introduite de manières diverses dans les années 1920-30 dans le contexte de l'histoire de l'art (H. Wölfflin, E. Panofsky), de l'histoire des civilisations (O. Spengler), ou de l'histoire des formes symboliques (E. Cassirer). Tout nouveau style de pensée correspond, pour Fleck, au choix d'un nouveau genre de savoir considéré comme essentiel. Les différences dans le but visé et dans la nature du sens qui est transmis sont telles que Fleck parle d'intraductibilité entre des styles de pensée: «If a thought style is so far removed from ours as this, no common understanding is any longer possible; words cannot then be translated and concepts have nothing in common with ours» (Genesis, p. 139). Le style de pensée des Temps Modernes se caractérise par exemple par un respect pour l'idéal de la vérité objective, de la clarté et de la rigueur, par la conviction que cet idéal sera atteint dans le futur, fût-ce asymptotiquement, et par une valorisation du sacrifice personnel à la tâche de la connaissance (l'individu doit s'effacer devant la découverte des lois de la nature): «It is expressed as a common reverence for an ideal - the ideal of objective truth, clarity and accuracy. It consists in the belief that what is being revered can be achieved only in the distant, perhaps infinitely distant future; in the glorification of dedicating oneself to its service; in a definite heroworship and a distinct tradition. This was the keynote of the common mood in which the thought collective of natural science lives its life» (ibid., p. 142).

7 L. Fleck, Genesis, p. 38. Cette épistémologie comparative a une autre intention polémique, puisque Fleck veut également s'attaquer aux théories de la science conventionnalistes et positivistes, dont il critique le point de vue trop formel. Cf. ibid., pp. 9-10: «The adherents of all these formal points of view pay far too little, if any, attention to the cultural-historical dependence of such an alleged epistemological choice - the alleged convention (...). A stylistic bond exists between many, if not all, concepts of a period, based on their mutual influence ». Au lieu de dire que les raisonnements en science sont guidés par un libre choix rationnel de l'ordre de la convention, il faut dire, selon Fleck, qu'ils sont guidés par le mode de pensée qu'est le Denkkollektiv. Toute expérience au sens propre doit donc être comprise comme un état complexe de la formation intellectuelle, qui repose sur l'interaction entre celui qui connaît, le savoir qu'il possède déjà, et ce qu'il a encore à apprendre. Les éléments qui entrent en jeu dans le processus de connaissance sont irréductibles à la seule logique formelle: ce sont l'acquisition de talents et de savoirs-faire, l'accumulation d'un certain nombre d'observations et d'expérimentations, la capacité à former des 
Comment, dans ces conditions, chaque individu est-il affecté par le Pensercollectif? A lire Fleck, l'on constate qu'il s'agit de fait d'une infection, à la fois subreptice et envahissante, qui ne laisse à l'individu qu'une marge de manœuvre très réduite. Le Penser-collectif est en effet comme sédimenté en nous, il nous est presque invisible, de sorte que l'on tend toujours à reconnaître comme une réalité objective de l'expérience ce qui n'est en réalité que le résultat des pré-conditions du savoir ${ }^{8}$. Le Penser-collectif transmet à l'individu tous les outils de son apprentissage: concepts, méthodes, formulation des problèmes, organisation de la recherche selon telle ou telle division du travail, modes de préparation des phénomènes, bon usage des contraintes techniques, rhétorique des controverses, publications à plusieurs, structures hiérarchiques à l'intérieur des communautés ${ }^{9}$. Le savant, comme tout autre individu, pense donc selon une structure formelle qui s'avère de nature essentiellement sociale. En outre, il existerait une sorte d'accumulation primitive d'émotions tacitement partagées par la communauté et inscrites au fondement même des grands concepts scientifiques. Fleck affirme en effet que la connaissance est toujours liée au comportement émotionnel des individus - «Feeling, will and intellect all function together as an indivisible unit ${ }^{10}$ - et que ce que l'on appelle usuellement connaissance objective, ou pure, ou non subjective, est simplement une connaissance telle que son soubassement émotionnel est partagé par tous les membres du groupe. La neutralité du sujet connaissant devient une baudruche: «there is only agreement or difference between feelings, and the uniform agreement in the emotions of a society is, in its context, called freedom of emotions ${ }^{11}$.

concepts. Le passé survit en nous sous la forme des concepts que nous utilisons, sous la forme de la présentation même des problèmes et de la formulation des questions, sous la forme du langage ordinaire et des institutions (ibid., p. 20). Et cet état de choses est si complexe qu'il interdit toute approche systématique du processus de connaissance: «There is therefore no raison d'etre for any speculative epistemology» (ibid., p. 11); «Epistemology without historical and comparative investigations is no more than an empty play on words or an epistemology of the imagination» (p. $21)$; «I do not agree with the view that the sole or even most important task of epistemology consists in this kind of examination of the consistency of concepts and their interconnections within a system» (p. 22). L'épistémologie comparative sera donc une épistémologie non systématique, qui prendra en considération la pluralité des éléments qui entrent dans le processus de la connaissance et leur variation au cours de l'histoire.

8 L. Fleck, Genesis, p. 40: «Objective reality can be resolved into historical sequences of ideas belonging to the collective».

9 L. Fleck, Genesis, p. 42.

10 L. Fleck, Genesis, p. 94. Cf. aussi p. 49: «The concept of absolutely emotionless thinking is meaningless».

11 L. Fleck, Genesis, p. 49. Cela ne signifie pas que toutes les émotions soient équivalentes du point de vue de leur rôle cognitif. Il s'agit seulement d'apercevoir cet état de choses que, dans toute communauté, il existe des émotions partagées si fondamentales qu'elles entrent ainsi inaperçues dans le processus du jugement. Fleck mentionne le cas de la causalité : «The causality relation, for instance, was long regarded as purely rational, yet it was actually a relic of strongly emotive demonological ideas belonging to the collective» (ibid., p. 49). On peut également penser au cas du vide. L'on admet tacitement aujourd'hui que tel phénomène se manifeste de telle manière dans un vide reproduit en laboratoire. Le mot «vide» n'occasionne alors aucune émotion. Mais lorsque Galilée, Torricelli et Pascal font du vide, entre 1638 et 1646, un concept fondamental de la physique, la seule mention du terme suffit à bouleverser n'importe quel Jésuite. Un concept est de l'émotion apprivoisée. 
L'individu que décrit Fleck semble donc entièrement sous l'emprise du Penser-collectif. Son rôle dans la connaissance est faible, voire insignifiant, par rapport au développement propre des «forces sociales à l'œuvre dans le savoir». Le Penser-collectif est plus stable et plus cohérent que ce qu'on appelle l'individu, qui est toujours fait de pulsions contradictoires. Pris isolément, chacun de nous n'est d'ailleurs jamais ou presque jamais conscient du style de pensée dominant, qui exerce pourtant une pression absolument contraignante sur sa pensée. La conception des choses qui est caractéristique de la communauté et de ses «systèmes d'opinion» agit jusque dans la structure même du langage ${ }^{12}$. Réduit comme peau de chagrin, le rôle de l'individu dans la connaissance est seulement de «s'assurer des conséquences qui s'ensuivent de certaines préconditions ${ }^{13}$. En d'autres termes, l'individu enregistre, formule, contrôle, les implications entre certaines préconditions du savoir posées par le fonctionnement collectif de la pensée (les «connexions actives») et certains résultats d'expérience (les «connexions passives») qui forment ce que l'on considère comme réalité objective ${ }^{14}$. Qu'est-ce par exemple que voir un fait? C'est voir quelque chose et l'interpréter comme un fait. Or, dans ce processus le Penser-collectif fournit la totalité de la préparation et de la disponibilité intellectuelles pour acquérir telle façon particulière de voir et d'agir, plutôt que telle autre. Voir, en effet, ne se réduit pas à: avoir une perception visuelle; voir, c'est avoir la perception visuelle développée d'une forme (Gestaltsehen $)^{15}$. Un fait scientifique émerge d'abord parce qu'il se produit un signal de résistance dans la façon de penser originaire, puis parce que l'individu obéit à une contrainte bien définie, enfin parce qu'il dégage ainsi une forme qu'il croit dès lors percevoir directement. En dernière instance, le Penser-collectif indique donc à l'individu non seulement selon quels concepts et règles penser, mais même ce qu'il voit dans son expérience.

12 Pour plus de détail, cf. L. Fleck, Genesis, pp. 40-45.

13 L. Fleck, Genesis, p. 40: «Cognition therefore means, primarily, to ascertain those results which must follow, given certain preconditions. The preconditions correspond to active linkages and constitute that portion of cognition belonging to the collective. The constrained results correspond to passive linkages and constitute that which is experienced as objective reality. The act of ascertaining is the contribution of the individual».

14 Fleck appelle «connexions passives» ou «associations passives» des liens réguliers observés dans une certaine classe de phénomènes. Il appelle «connections actives » des associations suggérées, inventées, imposées par le fonctionnement collectif de la pensée. La distinction n'est pas toutefois une distinction de nature entre différents énoncés. Car tout énoncé qui se présente comme une connexion passive peut être considéré comme une connexion active si on l'envisage sous un autre point de vue (Genesis, pp. 47-48). En d'autres termes, il n'y a jamais de lecture neutre et objective de l'expérience, qui donnerait lieu ensuite à un travail de mise en ordre. Tout est culturel et historique, dans le processus de connaissance, et ce qui se présente à une époque donnée comme «réel», «objectif» ou «vrai» peut se manifester soit comme venant de l'empirique, soit comme une décision du collectif. Le rôle de l'individu est simplement de s'assurer qu'il existe bien un lien d'implication entre ce qui se présente comme connexions observées et ce qui se présente comme concepts unificateurs produits par la communauté.

15 Une description de l'opération qui consiste à voir un fait doit distinguer trois étapes, selon Fleck: (a) celle de la perception visuelle initiale et vague, ou de l'observation encore incomplète et inadéquate; (b) celle de la formation d'une interprétation, qui est conditionnée par le style; (c) celle de la perception visuelle développée, reproductible et stylisée de la forme. 
L'individu, pour tout dire, est un chaos. Comme il participe inévitablement de plusieurs modes de Penser-collectif en même temps, étant par exemple, membre de la communauté scientifique et aussi membre d'un parti politique, d'une classe sociale, d'une nation ou de telle ou telle société secrète, il peine à ajuster tout cela. Vivant dans plusieurs mondes symboliques, il reste imperméable en général aux contradictions, se réglant selon une uniformité de pensée du point de vue social qui est beaucoup plus forte que sa pensée logique, au point que même les contradictions logiques de cette pensée «sociale» n'atteignent même pas un niveau explicite $^{16}$.

Fleck procède ainsi en 1935 à une critique radicale des philosophies de la conscience et de toute problématique de la constitution de l'objet par un sujet transcendantal, au profit d'une compréhension de la connaissance comme fait social, presque comme «construction sociale» au sens des sociologues des sciences contemporains. En ceci il prend acte d'un mouvement qui connaîtra toute son ampleur dans les années 1960. En histoire des sciences, l'on supposera qu'il se produit des «mutations discontinues» de la pensée dont l'agent réel serait la communauté, et qui modifierait quelque chose que l'on appelle selon les auteurs cadre conceptuel, style de pensée, Weltanschauung, paradigme ou matrice disciplinaire, système d'énoncés. En philosophie, le corrélat de cette désindividualisation du processus de la connaissance sera la «crise du sujet». L'on critiquera les illusions de la philosophie réflexive selon lesquelles un Je constituerait en totalisations le temps, l'histoire, le monde, etc. Foucault, décidant à cette époque de faire plutôt une théorie des pratiques discursives qu'une théorie du sujet de la connaissance, écrira: «S'il est une approche que je rejette catégoriquement, c'est celle (appelons-la en gros, phénoménologique) qui donne une priorité absolue au sujet de l'observation, attribue un rôle constitutif à un acte et pose son point de vue comme origine de toute historicité - celle, en bref, qui débouche sur une conscience transcendantale $»^{17}$.

\section{B) Le retour de l'individu : science et ambitions personnelles}

Fleck remplace le sujet de la philosophie moderne par le Penser-collectif via une opération dont le résultat est qu'il n'y a plus d'individu à proprement parler: on jette le bébé avec l'eau du bain, l'individu avec le sujet transcendantal.

Dans la sociologie des sciences des dix ou quinze dernières années, il semble au contraire que l'on observe un certain retour de l'individu; mais ce retour lui est tellement dommageable qu'il ne faut peut-être pas s'en réjouir! De fait tout se passe comme si l'idée même d'une personnalitas moralis (Kant) était devenue exsangue. Dans beaucoup de conceptions actuelles de la science, l'individu, s'il a la chance de n'être pas réduit à une candeur toute huronienne, apparaît comme un «acteur» ambitieux et dominateur, engagé sans cesse dans des controverses où se jouent avant tout des relations de pouvoir.

Cette image, qui passe pour la seule «intelligente» parce que la seule à prendre en compte sans hypocrisie la réalité des mécanismes de domination

\footnotetext{
L. Fleck, Genesis, p. 110.

17 M. Foucault, Foreword to the English Edition, in M. Foucault, The Order of Things, Londres, Tavistock, 1970; traduit in Dits et Ecrits, t. II, Paris, Gallimard, 1994, p. 13.
} 
immanents à chaque groupe social, est associée à une conception plus générale de la connaissance qui en fait une négociation sociale de preuves. Considérons sous ce rapport le programme dit «fort» de la sociologie des sciences contemporaine ${ }^{18}$. Comme il ne s'agit pas pour moi ici de débattre du détail des thèses avancées, je ferai référence seulement à la synthèse de ces thèses qui a été proposée en 1998 par D. Pestre ${ }^{19}$. La nouvelle histoire des sciences, écrit Pestre, décrit la science telle qu'elle se fait. Sous-entendu: elle ne s'attache pas, comme le faisait le positivisme logique, à la reconstruction rationnelle de la structure logique des théories physiques. Qu'est-ce que décrire la science telle qu'elle se fait? C'est d'abord, négativement, détruire un certain nombre d'illusions idéalistes. La science apparaîtrait, à tort, comme un savoir qui serait avant tout conceptuel, avec des démonstrations contraignantes, indépendantes de l'évolution des sociétés, un savoir muni d'une méthode propre, confiant dans la reproductibilité des résultats expérimentaux dans la nature et certain de dévoiler le sens du monde naturel. Détruire ces illusions signifierait alors, positivement, que l'on décrive la science avant tout comme une institution sociale et un ensemble de pratiques et de savoir-faire. Comme un champ où aucune solution n'est contraignante et où les solutions sont adoptées en fonction de la conception que se fait l'époque de l'intelligibilité et de la preuve. Comme une activité locale liée à l'état des techniques et de l'industrie dans telle zone géographique. Comme une activité qui n'a pas de méthode propre, mais qui évolue dans la complexité toujours contradictoire des modes de légitimation des savoirs. Comme une fabrique d'expérimentations qui sont des effets de théâtre, produits dans des espaces spéciaux. Enfin, comme un discours qui se targue de la caution d'une Nature elle-même muette, et qui ne semble universel que parce que l'institution le fait circuler comme tel.

Ces thèses reviennent donc à historiciser et à anthropologiser radicalement la notion de science. Il est outrecuidant de les rejeter d'un revers de main, comme le font beaucoup de scientifiques; il me semble qu'il faut plutôt les examiner soigneusement et les interpréter comme une modélisation possible d'une certaine situation historique, récurrente sous certaines conditions, du travail intellectuel. Mais ce qui m'importe ici est que la sociologie des sciences tend aujourd'hui sous cette inspiration à décrire l'individu d'une façon que je trouve non seulement sinistre, mais aussi fausse.

Peut-on donner des exemples précis de cette prétendue dégradation si fâcheuse de la personnalitas moralis une fois privée du transcendantal? Il y en aurait pléthore, fournis par la littérature constructiviste... J'évoquerai ici le cas, tout à fait singulier, de la façon dont l'on présente aujourd'hui les principaux fondateurs de la mécanique quantique comme des êtres cyniques qui auraient utilisé toutes les ficelles de pouvoir répertoriées afin de promouvoir leur interprétation

18 Ou «programme d'Edimbourg». En 1974, Barry Barnes publie un livre intitulé Scientific knowledge and sociological theory (Londres, Routledge), dans lequel il affirme le caractère socialement construit des critères du vrai et du faux. Barnes est ensuite suivi dans cette voie par de très nombreux «nouveaux sociologues» comme D. Bloor, H. Collins, K. Knorr-Cetina, M. Lynch.

19 Voir D. Pestre, Les sciences et l' histoire aujourd' hui, in «Le Débat, Comment écrire l'histoire des sciences? Catherine Chevalley, Pierre Jacob, Gérard Jorland, Dominique Pestre», no 102, novembre-décembre 1998. Voir aussi dans le même numéro les articles de Gérard Simon et de Gerald Holton,. 
de la théorie. C'est ce que fait, par exemple, l'un des livres récents importants publié sur la formation de la mécanique quantique à la fin des années 1920, celui de M. Beller, Quantum dialogue. The Making of a Revolution ${ }^{20}$. Il s'agit d'un livre bien informé sur le plan documentaire, qui est le résultat de longues années de travail et qui est reconnu pour tel. Ce livre se situe dans un courant qu'on pourrait appeler sociologico-communicationnel, puisque son but principal est de montrer «en quoi la science est enracinée dans la conversation» (ibid., p. 2), qui serait le véritable lieu naturel d'un «tourbillon de concepts et d'émotions» (conceptual and emotional turmoil, ibid.) propice à l'élaboration du savoir: «Je soutiens, écrit l'auteur, que le dialogue est l'élément sous-jacent à la créativité scientifique» (ibid.). Le recours à l'idée de «flux dialogique» permettrait de contourner les notions massives usuelles de l'histoire des sciences: cadres conceptuels, schèmes ou paradigmes (ibid., p. 3). Il permettrait également d'introduire directement les stratégies rhétoriques dans le processus de construction d'une théorie: récits des vainqueurs, reconstitutions biaisées du passé, techniques de persuasion, argument de l'inévitabilité de la théorie, etc., auraient servi à établir dogmatiquement le pouvoir de la mécanique quantique acausale de Copenhague (ibid., pp. 10-11). Dès lors la voie est ouverte pour traiter Niels Bohr de «poète de la physique » (en un sens péjoratif) au nom de son usage « des analogies affectives, des associations subjectives, des harmonies, du langage ordinaire...» (ibid., pp. 12-13). La mécanique quantique version Copenhague aurait ainsi reposé dès ses débuts sur «les idées douteuses et pauvrement exposées de Bohr, qui dépassait ici son domaine de compétence» (ibid., p. 270) $)^{21}$.

Il n'y a, à vrai dire, rien de notoirement nouveau dans l'idée de transformer Bohr en Saint Sébastien de la Nouvelle Religion. D'autres flèches lui avaient été envoyées, tout aussi acérées, par K. Popper en 1982, qui écrivait sans aucune argumentation que l' «orthodoxie quantique» présentait tous les caractères des partis totalitaires; ou par G. Lochak qui parle en 1992, dans sa biographie de L. de Broglie, du «manteau impérial de la théorie de la complémentarité», traite Bohr de «nouveau Bellarmin» et soutient qu'il s'agissait d'un «coup d'état»; ou encore par J. L. Heilbron en 1985, qui traite Bohr de «gourou» et décrit la philosophie de Copenhague comme «un mélange d'impérialisme et de résignation ${ }^{22}$. La diabolisation de Bohr remonte à vrai dire au milieu des années 1950, lorsque l'on s'est mis à employer les expressions d'Interprétation de Copenhague ou d'orthodoxie. C'est à ce moment que s'est peu à peu formée l'équation suivante:

20 M. Beller, Quantum dialogue. The Making of a Revolution, Chicago, The University of Chicago Press, 1999.

21 Je ne critique si ouvertement le travail de M. Beller que parce que, du fait même de son érudition, il me semble propre à accréditer une façon de présenter les choses avec laquelle je suis en désaccord. Il me semble qu'une toute autre interprétation du mythe doxographique de Bohr est possible, qui s'appuierait sur la notion de «fantastique transcendantale» introduite par P. Ricoeur; pour une tentative dans ce sens, voir C. Chevalley, Mythe et Philosophie. La construction de 'Niels Bohr' dans la doxographie, in Phûsis, Florence, L. Olschki, vol. XXXIV, fasc. 3, pp. 569-603.

22 Cf. resp. K. Popper, Quantum Theory and the Schism in Physics, Londres, Hutchinson, 1982; G. Lochak, Louis de Broglie, Paris, Flammarion, 1992, p. 138; J. L. Heilbron, The Earliest Missionaries of the Copenhagen Spirits, in «Revue d'Histoire des Sciences», vol. XXXVIII, no 3-4, 1985, pp. 195-230. 
N. Bohr $=$ BOHR $1927=$ Interprétation de Copenhague $=$ Standard View, équation qui suppose une série de simplifications drastiques, mais qui permet d'identifier de proche en proche le travail de Bohr de 1912 à 1927 à un seul texte écrit en 1927-28, puis à une orthodoxie dogmatique qui aurait uni Bohr, W. Heisenberg, W. Pauli, M. Born, P. A. M. Dirac, P. Jordan, J. von Neumann et bien d'autres, et enfin à ce qui est devenu l'interprétation dite standard vers $1970^{23}$. Dans les années 1960-70, plusieurs philosophes des sciences avaient aperçu cette situation: N. Hanson, M. Jammer, E. Scheibe, P. K. Feyreabend, E. McKinnon, D. Howard. Mais leur protestation souvent violente semble être restée inaudible, malgré leur très grand renom. Dans une entreprise dévastatrice et assez étrange de destruction de tout ce qui pourrait s'opposer à la vénération à l'égard d'Einstein, l'on a commencé à dire couramment que l'Interprétation de Copenhague s'était imposée de force, par le jeu des pressions sociales et du charisme de ses chefs. Cette hypothèse est par exemple soutenue avec vaillance par J. Cushing dans son livre Quantum Mechanics. Historical Contingency and the Copenhagen Hegemony, qui entend montrer qu'une chronologie historique différente aurait conduit à l'adoption du «programme causal» et non du programme dit de Copenhague ${ }^{24}$.

Faut-il prendre de tels jugements au sérieux? Cent objections ruinent la thèse selon laquelle Bohr, Heisenberg, Pauli ou d'autres auraient engagé par ambition personnelle l'interprétation de la mécanique quantique dans la direction d'une sorte de déchetterie pour concepts philosophiques. Il reste qu'aujourd'hui l'on affirme sans rougir que la prétendue «Ecole de Copenhague», qui n'existe en tant que telle que dans les esprits de ses adversaires des années 1950 et suivantes, aurait été un groupe de pression dès l'origine, qui se serait imposé par des moyens publicitaires et qui aurait fonctionné comme un Penser-collectif, dictant à tous comment manipuler, comment formaliser, comment interpréter, comment gloser.

Tout se passe alors comme si la sociologie devait à bon droit remplacer l'épistémologie, au nom de sa courageuse dénonciation des mécanismes de pouvoir à l'œuvre chez les savants. L'image qui en résulte est fausse historiquement, elle fait bon marché de ce que Husserl appelait l'impulsion des problèmes, et de plus elle est triste, décourageante, moralisatrice et ennuyeuse. Somme toute, Simone Weil était bien plus drôle lorsqu'elle chargeait le «village des savants » tout entier, non pas au nom de la morale, mais au nom de la statistique: les savants? Pas pires que les autres groupes sociaux, disait-elle en substance, pas meilleurs non plus. Ou

23 Les Archives Bohr ont été ouvertes en 1985 et les principaux travaux d'histoire des sciences qui s'en sont inspirés donnent une image très différente des choses; voir notamment les publications de O. Darrigol, S. Petruccioli, J. Faye, A. Pais, H. Folse, J. Honner, D. Murdoch, F. Aaserud.

24 J. Cushing, Quantum Mechanics. Historical Contingency and the Copenhagen Hegemony, University of Chicago Press, 1994, p. xi: «A reordering of historical factors could reasonably have resulted in the causal program having been chosen over the Copenhagen one (...). The Copenhagen group had the talent organization and drive to carry the day in establishing the hegemony of its view ». Cushing fait de nombreux anachronismes, comme celui de définir l'Interprétation de Copenhague comme centrée de manière non problématique autour du problème de la réduction du paquet d'ondes (ibid., p. 17), alors que Bohr s'opposait explicitement à J. von Neumann sur ce point dans un article de 1938. Il critique la dérive de Bohr vers l'ontologie («Bohr's slip from epistemology to ontology », in ibid, p. 25), alors que Bohr ne cesse de s'opposer à toute ontologisation de nos représentations. Il mentionne comme allant de soi l'acceptation tacite par Bohr de critères positivistes (p. 26) ou opérationnalistes (p. 28), ce qui ne résiste pas à l'examen. 
bien Nietzsche, lorsqu'il comparait les hommes de science au sens large (savants et hommes de lettres) à de petits mécanismes d'horlogerie ou à des miroirs sans autre joie que celle de refléter ${ }^{25}$.

Les individus ont bien retrouvé un rôle dans la sociologie récente des sciences. Mais ce rôle les a réduits à des acteurs ambitieux paradant sur la scène de théâtre des controverses, des coups bas, des financements odieux, des compromis abjects. N'est-il pas possible de formuler les choses autrement, sans pour autant tomber ni dans les naïvetés de notre ancienne foi métaphysique dans la science, ni dans les excès d'une description qui rend l'individu entièrement passif et soumis au Penser-collectif, ni enfin dans une critique dénuée de probité philologique, lourde, et moralisatrice de la méchanceté cachée des héros de la pensée?

\section{SITUATIONS DE CONTRADICTION ET TECHNIQUES INDIVIDUELLES D'ÉCART}

Dans Changing Order, H. Collins lui-même écrit que «le premier ingrédient de la recette pour changer d'ordre est un individu disposé à proposer une interprétation des données qui ait la potentialité de créer une certaine contradiction ${ }^{26}$. Je voudrais me situer à ce point dans la situation générale où quelqu'un est amené à dire Blanc là où tous les autres disent Noir, et poser la question suivante: que fait un individu confronté à un état de choses dont la communauté, soit par son attitude soit par ses modes de savoirs acquis, semble lui interdire l'intelligibilité? Une telle situation est fréquente dans l'histoire des sciences et j'en donnerai quelques exemples plus loin. Mais commençons par la considérer dans sa plus grande généralité formelle: «Je» dis quelque chose qui n'est pas entendu, ou qui est contredit, par tout le monde autour de moi. Qu'est-ce que je fais? Réponse: j'invente des techniques d'écart qui me sont absolument spécifiques et apparaissent en ce sens comme le produit d'un travail individuel commencé depuis toujours.

\section{A) Les outils interprétatifs de la communauté et le travail herméneutique de l'individu}

Pour la clarté des choses, je voudrais préciser en premier lieu en quel sens minimal je prends les termes de communauté et d'individu.

Une communauté peut d'abord être définie comme un lieu où l'objectivité se définit par l'accord intersubjectif d'un certain nombre d'individus qui parviennent

25 Cf. F. Nietzsche, Par-delà Bien et Mal, § 206-207. L'homme «objectif» est, dit Nietzsche, quelqu'un qui est «travailleur, qui prend docilement sa place dans le rang, dont les talents et les besoins sont mesurés et réguliers, qui sait flairer ce lopin de vert pâturage sans lequel on ne peut travailler tranquille...». L'homme objectif est celui qui recherche «le rayon de soleil de la bonne renommée, une constante confirmation officielle de sa valeur». Il est «serein, non pas faute de détresse, mais parce qu'il n'a pas de doigts pour sa détresse, qu'il ne sait pas la manier »; «son âme de miroir, toujours attentive à rester bien lisse, ne sait plus affirmer ni nier ».

26 H. Collins, Changing Order, Chicago, University of Chicago Press, 1985, p. 51: «The recipe for changing order starts with an individual who is prepared to put forward an interpretation of data which has the potential to create some contradiction ». 
à se communiquer leur expérience dans un langage non ambigu, parce qu'ils acceptent les mêmes modes perceptifs, les mêmes formalismes et les mêmes grandes stratégies d'interprétation. C'était la conception de Bohr et de Pauli ${ }^{27}$. Un tel groupe n'est alors ni complètement rationnel, ni complètement irrationnel. Une communauté serait complètement rationnelle si elle ne se réglait que selon la logique interne des problèmes; or il est clair que les décisions des groupes sociaux sont le résultat de multiples contraintes, certaines intellectuelles, d'autres de réalisation ou d'influences. Une communauté serait à l'inverse complètement irrationnelle si elle se donnait toute entière à l'ambition, à l'argent, au pouvoir ou au caprice - mais il est clair là encore que les logiques spécifiques des problèmes et même des déontologies interviennent à l'occasion dans les décisions... Aussi faut-il considérer, plus raisonnablement, qu'une communauté est a-rationnelle. Elle se définit par l'adhésion du plus grand nombre de ses membres à certains outils ou schèmes interprétatifs. De la même manière, un tel groupe ne saurait être ni méchant ni bon, ni pervers, ni honnête. Les communautés savantes ne font en général d'éthique qu'en Comités. Les outils interprétatifs sur lesquels s'accorde une communauté peuvent inclure aussi bien un marteau (une communauté peut décréter que Un tel est «fou»), qu'un tournevis (pour visser une explication-type dans la tête de ses membres). Une communauté prend des décisions politicardes ou scientifiques, publicitaires ou intellectuelles, mais dans tous les cas elle agit selon les grandes orientations interprétatives au nom desquelles elle s'est organisée. Les schèmes d'interprétation qui sont dominants dans une communauté ne sont intrinsèquement ni rationnels ni irrationnels, ni «éthiquement corrects » ni scandaleux. Ils sont simplement momentanément acceptés.

Soit donc une communauté a-rationnelle et a-morale, définie de façon minimale par l'adhésion d'un certain nombre de gens à des outils interprétatifs bien définis à tel et tel moment. Comment maintenant concevoir l'individu? J'admettrai ici que l'individu est toujours déjà engagé dans l'interprétation. Tout lui vient de l'expérience, mais en un sens particulier: l'expérience n'est pas seulement l'expérience de qui est présent-sous-la-main, de la chose subsistante. Elle est expérience de l'ambigu - ce qui explique que l'on peine tant à transmettre l'expérience. Elle est un travail incessant de l'interprétation, un processus herméneutique qui est une création continuée de nos «mondes». Douter où il faut, s'assurer où il faut, se soumettre où il faut, comme le disait Pascal, c'est savoir jouer avec cette ambiguïté. L'ambiguiité assure le renouvellement de la connaissance, qui vient toujours d'un afflux d'empirique inintelligible et d'expériences incohérentes. Elle se réduit - par la partition des régions d'expérience et la constitution de nouveaux langages efficaces -, puis se transporte ailleurs et recommence à soumettre l'individu à l'épreuve de s'affronter comme impuissant ou ignorant.

27 Cf. W. Pauli, Phänomenon und physikalische Realität (1957), in Physik und Erkenntnistheorie, Braunsweig, Vieweg, 1961; rééd. 1984 avec une introduction de K. von Meyenn, p. 95 (trad. anglaise: Writings in Physics and Philosophy, Berlin et New York, Springer, 1994, p. 129): «Je m'accorde avec Bohr dans l'opinion que l'objectivité d'une explication scientifique de la nature devrait être définie aussi libéralement que possible: toute manière de voir (jede Betrachtungsweise) que l'on peut transmettre à d'autres, que d'autres, ayant les connaissances préliminaires indispensables, peuvent comprendre et appliquer à leur tour, et au sujet de laquelle nous pouvons parler avec les autres, devra être dite objective». 
Un tel individu est par conséquent lui aussi, pris sous sa forme générale abstraite, a-rationnel et a-moral. Il n'est rationnel que dans des régions d'expérience bien délimitées, et que s'il dispose d'outils bien affutés qui ont déjà fait leurs preuves. C'est le cas, par exemple, en physique, en mathématiques, en logique; c'est même le cas souvent dans l'expérience amoureuse, pour peu que l'on observe que la plupart des comportements y sont régis par la règle de la répétition d'expériences littéraires ou cinématographiques. Quant à la personnalitas moralis, elle ne se structure facilement que sous la contrainte des vieux thèmes religieux, qui restent jusqu'à présent les recueils de règles les plus riches, les plus nombreux, et les plus précis que nous ayons. Notre époque est, pour le reste, indigente. Nous n'avons, dans l'université, qu'une vieille morale laïque et positiviste fabriquée il y a plus d'un siècle; nous n'avons, dans l'amour, que des variations lasses sur Sade, Miller, ou «Casablanca»; et nous n'avons, dans la politique, qu'un plaisir cathartique du théâtre, où les feux des projecteurs ne fonctionnent que pour les despotes (éclairés) qui ont leur chaise sur la scène! Comment s'étonner que nous ayons aujourd'hui quelque difficulté à penser l'individu?

En réalité, nous sommes à nous-mêmes ce qu'il y a de plus étranger, dit Nietzsche. Pour nous connaître, il ne faut donc pas chercher à nous re-connaître dans du déjà vu, déjà rencontré, déjà écrit, pensé, représenté: car re-connaître, ce n'est que se rassurer. Connaître exige plutôt d'aller vers ce que l'on ne reconnaît pas, et qui semble étranger, anormal, inintelligible. C'est d'ailleurs ce que font les physiciens, dit encore Nietzsche ${ }^{28}$. Pour se poser cette question si simple, «qu'aije envie de dire, de faire, de comprendre, de sentir?», avec l'espoir d'y répondre, il convient de se traiter soi-même comme une expérience que l'on met des années à monter, dont l'on peaufine les détails et dont l'on interprète prudemment les résultats statistiques. Je voudrais maintenant proposer l'ébauche d'une description de certaines techniques individuelles de compréhension de l'inintelligible, qui conduisent à ce que j'appellerai par un néologisme des «automathématismes »: des automatismes que l'on invente et que l'on s'enseigne à soi-même.

\section{B) Deux exemples : J. Kepler (1604); N. Bohr (1913)}

Mes exemples seront pris dans l'histoire des sciences. Je ne poserai aucun principe général de méthode susceptible de faire pendant aux quatre principes de causalité, de symétrie, d'impartialité et de réflexivité revendiqués par certains sociologues des sciences. Nietzsche parlait du gris de tout ce qui est attesté par les documents, ce que l'on peut vraiment établir et ce qui a réellement existé, bref, tout le long texte hiéroglyphique, laborieux à déchiffrer, du passé de la morale humaine. Foucault reprenait ce gris à son compte: La généalogie est grise; elle est méticuleuse et patiemment documentaire. Elle travaille sur des parchemins embrouillés, grattés, plusieurs fois réécrits ${ }^{29}$. Ce minimalisme méthodologique, auquel je souscris, n'est évidemment pas une revendication naïve de neutralité. Il vise plutôt à laisser la voie libre à une adaptation de la méthode aux objets, dans un «scepticisme systématique à l'égard de tous les universaux anthropolo-

\footnotetext{
F. Nietzsche, Le Gai Savoir, § 355.

29 Resp. F. Nietzsche, Généalogie de la morale, Avant-propos de 1887, § 7, et M. Foucault, Nietzsche, la généalogie, l' histoire (1971), in Dits et Ecrits, t. II, Paris, Gallimard, 1994, p. 137.
} 
giques $»^{30}$. Il faut partir non pas du sujet constituant de la phénoménologie, ni du sujet constitué des marxismes, mais des pratiques concrètes en tant que modes d'agir et de penser.

Gris des documents et analyse des pratiques concrètes. J'évoquerai d'abord l'exemple de la refondation de l'optique par Kepler en 1604, dans un livre touffu intitulé Ad Vitellionem Paralipomena (ce qui voulait dire «Compléments au manuel de Witelo») $)^{31}$. Avant ce livre, l'optique n'est pas une théorie autonome: elle est soit une partie de l'astronomie, soit une branche de la géométrie, soit un terrain d'expériences curieuses pour la magie naturelle, soit une technique picturale, soit encore une métaphysique de la lumière. En 1604, Kepler établit l'optique comme un savoir distinct, il donne la première explication correcte de la vision en montrant comment se forme l'image rétinienne à l'issue de la traversée des humeurs de l'œil par les faisceaux lumineux, il donne la première théorie des lentilles minces et des lunettes, et il formule une loi correcte de la réfraction pour les petits angles. Le livre de Kepler n'est pas seulement le début de l'optique moderne. Il ouvre aussi la voie à la loi des orbites elliptiques des planètes énoncée dans l'Astronomie nouvelle de 1609, c'est-à-dire à ce que A. Koyré appelait la fin du privilège du cercle; il ouvre la voie à la géométrie projective de Desargues et Pascal; et il ouvre la voie au sens nouveau que prend chez Descartes la référence à la métaphore de la vision dans la connaissance. Mais ce livre ne correspond pour autant en rien à la façon de penser usuelle. Kepler s'agrippe d'abord à une anomalie que Tycho Brahé trouvait pour sa part tout à fait explicable ${ }^{32}$. Il s'obstine dans l'étude de cette anomalie. Il fait l'hypothèse philosophiquement fondamentale que la bizarrerie des phénomènes observés tient à la nature de l'instrument utilisé (une forme de camera obscura). Il reprend à zéro l'analyse de la formation des images. Il puise dans les livres de magie naturelle les résultats des savoirs populaires sur les images, qu'il confronte au savoir anatomique tout nouveau de ses amis médecins. Il réfute la théorie aristotélicienne point par point dans un texte implacable de dix pages. Il se débarrasse de ses collègues à coups de grands moments lyriques souvent désopilants où il cite les poètes grecs et latins. Il utilise toutes les ressources des procédés de réfutation qu'il avait mis en pratique un peu auparavant dans l'Apologie contre Ursus. Il rit, il se moque, il se met en colère, il explicite toutes ses démarches erronées successives. Il reprend des techniques perspectives de Dürer et des résumés de théologie aux Néo-Platoniciens. Enfin il invente une langue nouvelle, en redéfinissant par exemple la distinction entre Imago et Pictura, ou en introduisant les concepts modernes de Foyer et de Convergence.

30 M. Foucault, Michel Foucault (1984), in Dits et Ecrits, t. IV, Paris, Gallimard, 1994, pp. 634-635.

31 J. Kepler, Ad Vitellionem Paralipomena, quibus Astronomiae Pars optica traditur, Francfort, 1604; trad. française et introduction: J. Kepler, Les fondements de l'optique moderne. Paralipomènes à Vitellion, Paris, Vrin, 1980.

32 Le point de départ de Kepler est le problème de la mesure des éclipses dans les «chambres noires » - en général les combles d'une église dont le toit était privé d'une tuile pour ménager une ouverture. Dans l'éclipse solaire de juin 1600, les observations montrent qu'il se produit une diminution du diamètre de la Lune tel qu'il est observé au moment de l'éclipse dans un dispositif de chambre noire, par rapport au diamètre de la pleine Lune. Tycho Brahé suggère que le phénomène s'explique par une dilatation périodique de la Lune. Kepler fait a contrario le geste décisif de mettre en question le dispositif expérimental lui-même et il propose de dire qu'il ne s'agit pas d'un phénomène réel, mais d'une particularité de sa représentation. 
Que fait Kepler en 1604? Il se donne à comprendre quelque chose d'inintelligible. Et pour cela il invente un comportement cognitif nouveau - un nouveau style et un nouveau langage. Il contredit le savoir de la communauté non pas au chaud de l'intérieur de ce savoir, mais en brisant son organisation interne. Il ne s'agit pas pour lui d'énoncer une proposition ou un groupe de propositions qui contredisent le Penser-collectif sur un point. Ce que Kepler fait concrètement est qu'il introduit une pagaille aussi complète que possible avant de réordonner le savoir existant avec des principes et des mots différents.

Cet exemple est-il exceptionnel et isolé? Absolument pas. En dépit de l'apparent quadrillage méthodologique que l'on doit aux interventions de Newton ou de Laplace, il existe bien d'autres exemples de démarches tout à fait semblables à celles de Kepler. C'est selon des procédés très semblables que Bohr introduit en 1913 la première théorie utilisable pour le comportement de l'électron dans l'atome d'hydrogène, dont E. Cassirer a pu dire qu'elle était «le point archimédien dont on avait tant besoin $»^{33}$. S'obstiner sur l'anomalie: celle que constituaient les données de la spectroscopie et l'instabilité mécanique de l'atome de Rutherford. Reformuler la question de la représentation de la structure de l'atome en faisant fi de l'usage antérieur des concepts canoniques: Bohr utilise deux notions de fréquence et il introduit un concept-monstre, celui d' «état stationnaire $»^{34}$. Pratiquer une totale liberté des hypothèses: Bohr suppose, en contradiction avec les théories électromagnétiques, que les électrons sont en mouvement sur des orbites fermées autour du noyau et que dans ce mouvement, ils n'émettent pas de rayonnement, de sorte que toute émission ou absorption d'un rayonnement par l'atome apparaît comme l'effet d'une transition quantique opérée par l'électron d'une orbite stationnaire vers une autre. Rire, se moquer: «Je n'essaie nullement de donner ce qu'on appellerait ordinairement une explication; rien n'a été dit au sujet du pourquoi et du comment la radiation est émise», écrit Bohr en $1914^{35}$. Tomber malade (ou, comme Pauli, sombrer momentanément dans l'alcool). S'acharner à réfuter point par point les hypothèses les plus tacites du savoir antérieur pendant des années. Changer de références, avoir recours à la littérature - au Faust de Goethe, comme tout le monde ${ }^{36}$ - à la peinture - au cubisme et à Picasso - et à la philosophie. Les physiciens qui fabriquaient la mécanique quan-

33 N. Bohr, On the Constitution of Atoms and Molecules, in «Philosophical Magazine», 26, 1913, pp. 1-25, 476-502 et 857-875. E. Cassirer donne un exposé remarquable des débuts de la théorie quantique dans Determinismus und Indeterminismus in der modernen Physik, Göteborg, 1936; trad. anglaise: Determinism and Indeterminism in Modern Physics, New Haven, Yale University Press, 1956.

34 Sur le concept d'état stationnaire et la forme générale de la théorie de 1913, cf. C. Chevalley, Introduction et Glossaire pour la réédition de N. Bohr, Physique atomique et connaissance humaine, Paris, Gallimard, 1991, resp. pp. 39-44 et 442-458.

35 N. Bohr, Om Brintspektret, in «Fysisk Tidsskrift», 12, 1914, pp. 97-114; trad. française dans N. Bohr, Les spectres et la structure de l'atome, Paris, Hermann, 1923, p. 18.

36 Goethe a accompagné tous les désespoirs de Bohr, de Heisenberg et de Pauli pendant les années 1920-30; en général, il leur servait toutefois à en rire! Une merveilleuse parodie du Faust est écrite et jouée à l'Institut de Copenhague en 1932, dans laquelle Pauli (Méphistophélès) essaie de vendre à l'agnostique Ehrenfest (Faust) l'idée d'un «neutrino sans masse» (Marguerite). On peut trouver une traduction française de cette parodie dans la traduction d'un livre de G. Gamow, Trente années qui ébranlèrent la physique, Paris, Dunod, 1968, pp. 137-182. 
tique avaient donné le nom de «la pagaille» ou «la supercherie» (Svindel) à leur attitude classique-quantique des années 1913-1924. Il s'agissait pour eux, de même que pour Kepler, de briser l'agencement interne des théories en usage en allant pas à pas et en faisant feu de tout bois. Il n'y avait aucune mystique révolutionnaire; seulement la volonté d'explorer, d'ajuster, de créer un nouveau langage.

Mon hypothèse est que comprendre, c'est inventer.

\section{C) S'enseigner à soi-même de nouveaux automatismes}

De ce comportement d'exploration, d'ajustement et de création, il est possible à mon avis de constituer une typologie. Il existe en effet des «techniques individuelles d'écart» à l'égard du savoir antérieur qui paraissent relativement invariantes si on les considère dans leur généralité abstraite. Ces techniques d'écart sont multiples: accepter de voir un signal de résistance - localiser l'inintelligible en aggravant les contradictions - faire une «critique logique» systématique de la validité des concepts - réfuter une à une toutes les solutions classiques - changer de point de vue, en repartant d'ailleurs - introduire d'autres modes d'agencement empruntés à la peinture, à la philosophie, à la littérature, à la musique - et changer le sens des mots. Certaines de ces étapes ont été décrites par tel ou tel auteur. Mises ensembles, elles suggèrent de considérer le travail de l'individu comme l'invention d'un moyen de supporter, de tourner ou d'éliminer l'inintelligible, plutôt que comme l'obéissance à des règles communautaires. Si l'on se tourne vers la philosophie, l'exemple le plus spectaculaire de telles techniques individuelles d'écart reste celui de Nietzsche: je me suis guéri moi-même, disait-il, des frissons et des angoisses de la solitude, auxquels toute absolue différence de vue condamne celui qui en est affligé, en inventant des « esprits libres » pour me tenir compagnie. Des «esprits libres » de ce genre, il n'y en a pas, il n'y en a jamais eu - mais j'avais alors, comme j'ai dit, besoin de leur société, pour rester de bonne humeur parmi des humeurs mauvaises (isolement, exil, acedia, inactivité): comme de vaillants compagnons et fantômes, avec lesquels on babille et l'on rit, quand l'on a envie de babiller et de rire, et que l'on envoie au diable, quand ils deviennent ennuyeux en remplacement, faute de mieux, des amis manquants $\gg^{37}$.

Comprendre, c'est inventer. Et ce sont les individus qui font ce travail. Précisément parce que la démarche ne suit aucun plan préordonné, aucune méthodologie préalablement acquise dans la communauté, elle dérive de ce que chaque individu est lui-même. Elle lui est spécifique ${ }^{38}$. Affronter la contradiction ne permet de «changer l'ordre» que lorsque celui qui soutient la contradiction a l'obstination, le culot, la rigueur, la volonté d'aller pas à pas, la possibilité de changer de point de vue, et l'attention à la langue. Il faut des automathématismes : des automatismes que l'on invente et que l'on s'enseigne à soi-même. La stylisation d'une conduite, la fabrication d'un langage.

37 F. Nietzsche, Humain trop humain, Avant-propos de 1886, § 2.

38 Le sommet de la politique est évidemment de s'arroger le monopole des techniques d'écart, en disant aux individus non pas tant ce qu'ils doivent penser, acheter, etc., mais ce qu'ils doivent critiquer, refuser d'acheter, etc. 


\title{
III. LA CONNAISSANCE COMME MODE DE SUBJECTIVATION ET PRATIQUE DE SOI
}

J'en viens pour finir à l'idée que si l'on veut pouvoir parler de l'individu, dans une situation où le Cogito est épuisé et où l' «acteur» sociologique se comporte de façon éhontée, il faut généraliser philosophiquement cette seconde figure du sujet que livre l'histoire des sciences. Pour cela, je voudrais faire une référence à Foucault, plus exactement aux écrits des dix dernières années de la vie de Foucault, dans lesquels il tentait de réintroduire en philosophie les notions de gouvernement de soi et des autres, de pratique de soi, et d'art de l'existence.

\section{A) Le postulat de Foucault : l'optimisme absolu quant à l'individu}

Il y avait chez Kant un optimisme de la communauté, au sens où Kant affirmait que l'humanité doit en principe se conduire rationnellement, non pas au niveau de l'individu, mais au niveau de l'espèce - en quelque sorte statistiquement. Lorsque Kant explique, dans l'Idée d'une histoire universelle au point de vue cosmopolitique, que l'individu n'est pas lui-même un être rationnel, ce n'est en effet que pour sauver le navire de l'humanité toute entière: «Etant donné que le philosophe ne peut supposer chez les hommes et dans leur jeu aucun dessein personnel raisonnable, il lui faut chercher s'il ne peut découvrir dans la marche absurde des choses humaines un dessein de la nature à partir duquel il serait au moins possible, à propos de créatures qui procèdent sans plan personnel, une histoire selon un plan déterminé de la nature ${ }^{39}$. L'humanité, chez Kant, est raisonnable, mais en tant qu'espèce, et la seule chose que l'on puisse espérer est qu'en «considérant globalement le jeu de la liberté du vouloir humain » l'on y découvre un cours régulier, malgré le «tissu de folie, de vanité infantile, souvent même de méchanceté et de soif de destruction puériles » (ibid.) que l'on voit exposés dans les faits et les gestes des hommes. C'était là, du moins, pour Kant, le dernier recours du philosophe désespéré.

Foucault ne croit en aucun plan caché de la nature ni même des hommes. Mais il croit dans la puissance d'agir de l'individu. Dans un entretien avec D. Trombadori, en 1978, Foucault énonce un extraordinaire «postulat d'optimisme absolu» s'agissant des gens :

\begin{abstract}
Les gens ont atteint l'âge de la maturité politique et morale. Il leur revient de choisir individuellement et collectivement. (...) Quand j'étudie les mécanismes de pouvoir, j'essaie d'étudier leur spécificité; rien ne m'est plus étranger que l'idée d'un maître qui vous impose sa propre loi. Je n'admets ni la notion de maîtrise, ni l'universalité de la loi. Au contraire, je m'attache à saisir des mécanismes d'exercice effectif du pouvoir; et je le fais parce que ceux qui sont insérés dans ces relations de pouvoir, qui y sont impliqués, peuvent, dans leurs actions, dans leur résistance et leur rébellion, leur échapper, les transformer, bref ne plus être soumis. (...). Il y a mille choses à faire, à inventer, à forger (...). De ce point de vue, toute ma recherche repose sur un postulat d'optimisme absolu ${ }^{40}$.
\end{abstract}

39 E. Kant, Idée d'une histoire universelle au point de vue cosmopolitique, in Euvres philosophiques, t. II, Paris, Gallimard, 1985.

40 Entretien avec Michel Foucault (1978), in «Il Contributo», 1, 1980, pp. 23-84; rééd. in M. Foucault, Dits et écrits, t. IV, Paris, Gallimard, 1994, pp. 41-95; ici p. 93. 
Que fait-on en philosophie avec un postulat? On fait la même chose que les mathématiciens lorsqu'ils «ajoutent un axiome» ou que les physiciens quand ils inventent des choses comme les quanta de lumière ou les états stationnaires d'un électron: faute de pouvoir argumenter son attitude, on se contraint soi-même à changer de point de vue par un coup de force. Avec son postulat d'optimisme absolu Foucault a recentré toute la philosophie et la politique, au début des années 1980, sur l'individu: c'est, dit-il, «une tâche urgente, fondamentale, politiquement indispensable, que de constituer une éthique du soi, s'il est vrai qu'après tout il n'y a pas d'autre point, premier et ultime, de résistance au pouvoir politique que dans le rapport de soi à soi $\gg^{41}$.

\section{B) Gouvernement de soi et des autres}

Comment alors définir l'individu? Certainement pas de manière abstraite et formelle. Les «gens», ce sont des individus pris un à un, chacun spécifique. L'individu n'est plus pour Foucault ni un Cogito cartésien, ni un «Je pense » kantien, ni une conscience transcendantale husserlienne, ni un être anthropologique. A partir de la fin des années 1970, Foucault pense en outre que les «systèmes anonymes d'énoncés » qu'il décrivait dans L'Archéologie du savoir et dans L'Ordre $d u$ discours ne suffisent pas à remplacer le sujet constituant de la philosophie moderne. Le sujet dont Foucault élabore une herméneutique dans ses derniers cours du Collège de France est, si l'on veut, un sujet éthique. Mais c'est un sujet éthique en un sens particulier. C'est un sujet constamment actif, constamment en train d'inventer ou de styliser des conduites, et nullement un sujet-assujetti à une loi morale. Il ne s'agit pas pour Foucault d'introduire subrepticement un nouveau code moral pour guider la subjectivité, ce code fût-il étayé sur une bonne théorie philosophique bien rigoureuse. L'individu est dit éthique dans la mesure où il se rend lui-même sujet de ses actes dans ses pratiques (du savoir, du plaisir, de la politique), dans la mesure où il stylise une conduite, où il développe un art de l'existence.

Dans l'Introduction de L'Usage des plaisirs, Foucault distingue trois sens du mot «morale»: le code dominant dans tel groupe à telle époque; le comportement effectif des individus; et enfin, au seul sens qui l'intéresse, la manière dont chacun détermine pour lui-même une «forme de subjectivation $»^{42}$. L'histoire des formes de subjectivation des individus dessine l'histoire des modes de rapport à soi, de pratiques de soi, de techniques de soi (connaissance, examen, aveu, trans-

41 M. Foucault, L'Herméneutique du sujet. Cours du Collège de France, 1981-1982, Paris, Gallimard/Seuil, 2001, p. 240 (Cours du 17 février 1982).

42 M. Foucault, L'Usage des plaisirs, Paris, Gallimard, 1984, p. 32. Une forme de subjectivation comporte ce que Foucault nomme la détermination de la substance éthique, puis le mode d'assujettissement à la règle choisie, puis le genre d'élaboration éthique que l'on effectue sur soi-même, enfin la téléologie de l'action. Dans l'article de synthèse que Foucault écrit sur son œuvre en 1984 (cf. note 30), il décrit son «histoire critique de la pensée» comme l'étude des modes de subjectivation et des modes d'objectivation qui scandent les relations entre sujet et objet, et il ajoute qu'il a «maintenant entrepris, toujours à l'intérieur du même projet général, d'étudier la constitution du sujet comme objet pour lui-même: la formation des procédures par lesquelles le sujet est amené à s'observer lui-même, à s'analyser, à se déchiffrer, à se reconnaître comme domaine de savoir possible» (ibid., in Dits et écrits, t. IV, p. 633). 
formation, purification, plaisir, etc.). Ou encore: elle est l'histoire même du «gouvernement », sous condition que, là encore, l'on redéfinisse le terme de gouvernement. Car il faut appeler "gouvernement», selon Foucault, non plus une instance exécutive quelconque, mais, au sens ancien du terme, toutes les techniques de conduite des individus ou de certains groupes spécifiés comme les enfants, les familles, les prisonniers, etc. Le «gouvernement» inclut les modes de l'éducation, les règles morales, les techniques du corps, du don, du partage, du châtiment, etc. Il est «l'ensemble des institutions et pratiques à travers lesquelles on guide les hommes depuis l'administration jusqu'à l'éducation ${ }^{43}$. Ou encore: «Il faut laisser à ce mot de gouvernement la signification très large qu'il avait au XVIe siècle. Il ne se référait pas seulement à des structures politiques et à la gestion des Etats; mais il désignait la manière de diriger la conduite d'individus et de groupes: gouvernement des enfants, des âmes, des familles, des communautés, des malades ${ }^{44}$.

Qu'est-ce donc que le gouvernement de soi, si l'individu que considère Foucault doit s'inventer lui-même ${ }^{45}$ ? C'est l'ensemble des techniques de pilotage par lesquelles chacun de nous tente de mener son existence. Foucault propose ici de redonner un sens à l'ancienne métaphore antique de la navigation. Nous ne savons plus naviguer, dit-il: «Quand on voit aujourd'hui la signification, ou plutôt l'absence de signification, qu'on donne à des expressions, pourtant très familières et qui ne cessent de parcourir notre discours, comme: revenir à soi, se libérer, être soi-même, être authentique, etc., quand on voit l'absence de signification et de pensée qu'il y a dans chacune de ces expressions aujourd'hui employées, je crois qu'il n'y a pas à être bien fier des efforts que l'on fait maintenant pour reconstituer une éthique du soi.» ${ }^{46}$ Or ce dont nous avons besoin est de nous constituer comme sujet, dans la plénitude du rapport à soi.

43 Même référence que note 40. Foucault livre à cette occasion une analyse de la situation historique dans laquelle se trouve à ses yeux la société occidentale: «Il me semble en effet qu'à travers la crise économique actuelle et les grandes oppositions et conflits qui se dessinent entre nations riches et pauvres (entre pays industrialisés et pays non industrialisés), on peut voir la naissance d'une crise de gouvernement. Par gouvernement, j'entends l'ensemble des institutions et pratiques à travers lesquelles on guide les hommes depuis l'administration jusqu'à l'éducation. C'est cet ensemble de procédures, de techniques, de méthodes qui garantissent le guidage des hommes les uns par les autres qui me semble aujourd'hui en crise, autant dans le monde occidental que dans le monde socialiste. (...). Je crois que, dans l'histoire de l'Occident, on peut trouver une période qui ressemble à la nôtre, même si évidemment les choses ne se répètent jamais deux fois, même pas les tragédies en forme de comédies: la fin du Moyen Age. Du XVe au XVIe siècle, on a observé toute une réorganisation du gouvernement des hommes, cette ébullition qui a amené le protestantisme, la formation des grands Etats nationaux, la constitution des monarchies autoritaires, la distribution des territoires sous l'autorité d'administrations, la Contre-Réforme, le nouveau mode de présence de l'Eglise catholique dans le monde. Tout cela a été une sorte de grand réaménagement de la manière dont on a gouverné les hommes tant dans leurs rapports individuels que sociaux, politiques. Il me semble que nous sommes à nouveau dans une crise de gouvernement. (...). Nous sommes peut-être au début d'une grande crise de réévaluation du problème du gouvernement».

44 M. Foucault, The Subject and Power, in H. Dreyfus et P. Rabinow, Michel Foucault, Chicago, The University of Chicago Press; traduit in Dits et Ecrits, t. IV, pp. 222-243; ici p. 237.

45 Foucault consacre une longue analyse au rôle assigné au philosophe comme guide. Se constituer soi-même requiert l'intervention d'un autre, qui est le philosophe, seul apte à vous faire comprendre la pikra anagkè, la nécessité amère de renoncer à ce que vous croyez vrai. Car le philosophe sait à la fois réfuter les opinions antérieures, et tourner l'esprit vers un autre côté.

46 M. Foucault, L'Herméneutique du sujet, op. cit. note 41, p. 241 (Cours du 17 février 1982). 
Se constituer comme sujet, cela veut dire refuser le sort du stultus, qui ne veut rien librement, qui ne veut rien absolument, qui ne veut jamais la même chose toujours. Cela veut dire aussi refuser d'être comme une toupie. La toupie tourne bien sur soi, mais comme il ne faut pas que nous nous tournions vers nous-mêmes. La toupie tourne sur soi à la sollicitation d'un mouvement extérieur. En tournant, elle présente diverses faces d'elle-même, toujours changeantes. Enfin, apparemment immobile quand elle tourne très vite, elle est en réalité toujours en mouvement. La sagesse demande le contraire. La sagesse demande de trouver au centre de soimême «le point auquel on se fixera et par rapport auquel on restera immobile ${ }^{47}$. La sagesse, enfin, demande que l'on apprenne à naviguer: que l'on sache se déplacer tout en restant solide à l'intérieur, que l'on sache se déplacer vers un but, que l'on sache que ce but est le véritable havre que l'on cherche, que l'on sache survivre à une traversée qui est toujours dangereuse. «La trajectoire vers soi a toujours quelque chose d'odysséen.» ${ }^{48}$

D'où l'idée d'une tâche incessante de fabrication de soi. Aux relations de pouvoir investies dans toutes les formes concrètes de gouvernement des conduites, chaque individu doit opposer sa liberté, comprise comme champ des possibles de ses conduites, et son choix de se gouverner soi-même, dans ce que Foucault appelle une «tâche politique incessante». Foucault est, comme Sartre, quelqu'un qui pose la liberté en postulat; mais il en tire moins la nécessité d'un engagement politique constant que celle d'un travail sur soi-même, qui rendra cet engagement politique significatif lorsqu'il deviendra nécessaire. C'est en ce sens que le souci de soi, l'askêsis, forme le lien entre vie des individus et vie de la Cité.

\section{C) Désenclaver la question de la connaissance}

A l'issue de ce déplacement de la question du sujet, il devient impossible de continuer à opérer une réduction de la question de la connaissance à l'une quelconque des disciplines dans lesquelles l'on a tenté de la fonder: métaphysique, théorie de la connaissance dans la tradition cartésienne et kantienne, phénoménologie transcendantale, sociologie ou anthropologie. C'est le schéma réductionniste lui-même qu'il faut abandonner. La connaissance n'a jamais une origine bien déterminée, pour un sujet défini lui-même de façon formelle et abstraite. Elle n'a pas, par exemple, son origine dans les semina scientiae, ni dans les pouvoirs de l'entendement pur, ni dans la peur, ni dans l'angoisse, ni dans de simples mécanismes neuronaux. La connaissance n'a de sens que comme l'une parmi d'autres des techniques de conduite que nous adoptons pour nous constituer nous-mêmes en sujets de nos actes.

Substituer l'individu qui se fabrique dans une pratique de soi aux concepts traditionnels de sujet connaissant, de sujet politique, de sujet esthétique, de sujet éthique, etc., ne revient donc pas à refonder la question de la connaissance dans une éthique déterminée. Ce serait un contre-sens total sur ce qu'il convient de

\footnotetext{
M. Foucault, L'Herméneutique du sujet, op. cit. note 41, p. 199.
}

48 M. Foucault, L'Herméneutique du sujet, op. cit. note 41, p. 238. On trouve une interrogation très semblable - comment empêcher le navire de tourner en rond sur lui-même? - chez Heisenberg en 1953; cf. W. Heisenberg, La nature dans la physique contemporaine (1955), Paris, Gallimard, p. 196; rééd. 2000, pp. 143-144. 
faire aujourd'hui. Ce qu'il faut faire aujourd'hui n'est pas inventer un nouveau code, mais reconstituer des individus capables de contredire, eux-mêmes et les autres, capables d'aimer, eux-mêmes et les autres, capables de "gouverner », euxmêmes et les autres. Il faut traquer l'insatisfaction du moi, qui détermine de manière plus ou moins directe toute violence: «Le mal revêt continuellement des formes innombrables, mais cette déformation a un résultat unique: l'insatisfaction du moi. La cause en est le déséquilibre de l'âme.» ${ }^{49}$ L'insatisfaction du moi se traque par l'art de voir, l'art de toucher, l'art de bouger, l'art de rire, l'art de fabriquer des «automathématismes ». La tâche de la philosophie est gigantesque, mais elle n'est plus ni grandiose-théorique ni universelle-systématique. Elle est de réenseigner la puissance d'agir, celle de sentir et celle de promettre.

Département de Philosophie

Université François Rabelais, Tours

49 Sénèque, De tranquillitate animi, XX. 